\title{
ARTICLE
}

Molecular Diagnostics

\section{Determination of breast cancer prognosis after neoadjuvant chemotherapy: comparison of Residual Cancer Burden (RCB) and Neo-Bioscore}

Enora Laas ${ }^{1}$, Julie Labrosse $\mathbb{D D}^{1}$, Anne-Sophie Hamy ${ }^{2}$, Gabriel Benchimol ${ }^{1}$, Diane de Croze ${ }^{3}$, Jean-Guillaume Feron ${ }^{1}$, Florence Coussy ${ }^{3}$, Thomas Balezeau ${ }^{4}$, Julien Guerin ${ }^{4}$, Marick Lae ${ }^{5}$, Jean-Yves Pierga ${ }^{3}$ and Fabien Reyal $\mathbb{D D}^{1,2,6}$

BACKGROUND: To compare RCB (Residual Cancer Burden) and Neo-Bioscore in terms of prognostic performance and see if adding pathological variables improve these scores.

METHODS: We analysed 750 female patients with invasive breast cancer (BC) treated with neoadjuvant chemotherapy (NAC) at Institut Curie between 2002 and 2012. Scores were compared in global population and by BC subtype using Akaike information criterion (AIC), C-Index (concordance index), calibration curves and after adding lymphovascular invasion (LVI) and pre-/post-NAC TILs levels.

RESULTS: RCB and Neo-Bioscore were significantly associated to disease-free and overall survival in global population and for triple-negative $B C$. RCB had the lowest AICs in every BC subtype, corresponding to a better prognostic performance. In global population, $\mathrm{C}$-Index values were poor for RCB $(0.66 ; \mathrm{Cl}[0.61-0.71])$ and fair for Neo-Bioscore $(0.70 ; \mathrm{Cl}[0.65-0.75])$. Scores were well calibrated in global population, but RCB yielded better prognostic performances in each BC subtype. Concordance between the two scores was poor. Adding LVI and TILs improved the performance of both scores.

CONCLUSIONS: Although RCB and Neo-Bioscore had similar prognostic performances, RCB showed better performance in BC subtypes, especially in luminal and TNBC. By generating fewer prognostic categories, RCB enables an easier use in everyday clinical practice.

British Journal of Cancer (2021) 124:1421-1427; https://doi.org/10.1038/s41416-020-01251-3

\section{BACKGROUND}

Neoadjuvant chemotherapy (NAC) is currently administered to patients with locally advanced breast cancers (BC), to $B C$ of poor prognosis (triple-negative and HER2-positive tumours, or $\mathrm{BC}$ with nodal involvement and/or high proliferation rates), or to early stage $\mathrm{BC}$ having an indication of systemic therapy. ${ }^{1,2}$ Beyond increasing breast-conserving surgery rates, ${ }^{3-5}$ NAC enables the evaluation of systemic treatments in vivo, thus making it theoretically possible to discontinue ineffective treatments. ${ }^{6,7}$ Response to NAC also carries important prognostic information. Indeed, patients with pathological complete response ( $p C R)$ after NAC were reported to have more favourable long-term outcomes, ${ }^{8,9}$ especially for HER2positive and triple-negative $\mathrm{BC}$ (TNBC). ${ }^{10}$ However, a minority of tumours reach $\mathrm{pCR}$ after NAC. Depending on definitions of residual disease (ypT0 ypN0 or ypT0/is, respectively), pCR rates vary from $13 \%\left(\right.$ IC $\left.95 \%{ }^{12-14}\right)$ to $22 \%\left(\right.$ IC $\left.95 \%{ }^{21,22}\right) .^{10}$

Hence, prognostic scores such as RCB (Residual Cancer Burden index) ${ }^{8}$ CPS (Clinical-Pathologic Scoring) ${ }_{1}^{11}$ CPS + EG (oestrogenreceptor (E) status and nuclear grade $(\mathrm{G}))^{11}$ and Neo-Bioscore ${ }^{12}$ were developed to classify BC patients into different prognostic risk categories after NAC. Thereby, patients considered as having a high risk of relapse can be candidates to further second-line treatments (TDM-1, Capecitabine) or clinical trials. Pathological variables such as lymphovascular invasion (LVI) and tumour-infiltrating lymphocytes' (TILs) may also have a prognostic value after NAC. ${ }^{13,14} \mathrm{LVI}$, defined as the presence of tumour cells in lymphatic or blood vessels, was identified as a risk factor of axillary and distant metastasis, ${ }^{15,16}$ associated to higher risks of node involvement, distant metastasis and death. ${ }^{13,17-19}$ High TILs levels on BC biopsy have been associated to high $\mathrm{pCR}$ rates in the neoadjuvant setting and to better outcomes in the adjuvant setting. ${ }^{20-23}$

Altogether, although RCB has been suggested as the preferred score, ${ }^{24}$ its prognostic performance has not yet been compared to more recent models such as Neo-Bioscore. Furthermore, despite growing evidence of their impact, whether LVI and/or TILs improve the prognostic performance of scores after NAC remains unknown.

The objective of the present study was to compare the main models that exist to refine prognosis after NAC by evaluating their prognostic performance in a large real-life cohort of BC patients,

\footnotetext{
${ }^{1}$ Department of Surgery, Institut Curie, Paris, France; ${ }^{2}$ Residual Tumor \& Response to Treatment Laboratory, RT2Lab, Translational Research Department, PSL Research University, INSERM, U932 Immunity and Cancer, Institut Curie, Paris, France; ${ }^{3}$ Department of Medical Oncology, Institut Curie, Paris, France; ${ }^{4}$ Data Office, Institut Curie, 26 rue d'Ulm, 75005 Paris, France; ${ }^{5}$ Department of Pathology, Institut Curie, Paris, France and ${ }^{6}$ University of Paris Descartes (Paris V), Paris, France

Correspondence: Fabien Reyal (fabien.reyal@curie.fr)

These authors contributed equally: Enora Laas, Julie Labrosse
}

Received: 1 April 2020 Revised: 2 December 2020 Accepted: 17 December 2020

Published online: 9 February 2021 
1422

and to determine whether adding pathological variables improved these scores.

\section{METHODS}

\section{Patients and tumours}

We analysed a cohort of 750 female patients with T1-3NxM0 invasive BC (NEOREP Cohort, CNIL declaration number 1547270) treated with NAC at Institut Curie between 2002 and 2012. The cohort included unifocal, unilateral, nonrecurrent, nonmetastatic tumours, excluding T4 tumours (inflammatory, chest wall or skin invasion). All patients included in the cohort received NAC. Patients were treated according to national guidelines. Approved by the Breast Cancer Study Group of Institut Curie, the study was conducted according to institutional and ethical rules concerning research on tissue specimens and patients. Informed consent from patients was not required.

Information on clinical and tumour characteristics were retrieved from medical health records.

ER, PR and HER2 positivity determination and treatment protocol are detailed in the supplemental material. Based on immunohistochemistry surrogates, pathological subtypes were defined as follows: tumours positive for either ER or PR and negative for $H E R 2$ were classified as luminal; tumours positive for $H E R 2$ were classified as HER2-positive BC; tumours negative for ER, $\mathrm{PR}$ and HER2 were classified as triple-negative BC (TNBC).

$\mathrm{RCB}$ and Neo-Bioscore were retrospectively reviewed by a single pathologist.

\section{Prognostic models}

pCR was defined as the absence of residual invasive cancer cells in the breast and axillary lymph nodes (ypT0/is +ypN0). ${ }^{10}$

Residual cancer burden (RCB) index was calculated using the web-based calculator (available on internet), ${ }^{25}$ by considering dimensions of the primary tumour, tumour bed cellularity and axillary nodal burden. ${ }^{8}$ RCB is composed of four categories: RCB-0 (complete pathologic response $=\mathrm{pCR}$ ), RCB-I (minimal residual disease), RCB-II (moderate residual disease) and RCB-III (extensive residual disease).

Neo-Bioscore ${ }^{12}$ derives from the CPS and CPS + EG scores. According to the American Joint Committee on Cancer (AJCC) guidelines, Neo-Bioscore is calculated by considering for each patient the pretreatment clinical stage and the post-treatment pathologic stage. ${ }^{26}$ Additional points are added in case of ERnegative disease, nuclear grade 3 tumours, and HER2-negative tumours. Neo-Bioscore is composed of eight categories (NeoBioscore 0-7), of increasingly poor prognosis.

LVI was defined as the presence of carcinoma cells within a finite endothelial-lined space (a lymphatic or blood vessel). Presence or absence of LVI was determined by unstained standard formalinfixed paraffin-embedded examination. Immunostaining with vascular markers was occasionally performed to rule out invasive carcinoma with shrinkage artefact. Data concerning LVI were extracted from pathologic records by two independent researchers.

TILs levels were evaluated on pretreatment core needle biopsies and post-NAC surgical specimens for the presence of mononuclear cells infiltrate (including lymphocytes and plasma cells, excluding polymorphonuclear leukocytes), following the international TILs Working Group recommendations. ${ }^{27}$ They were evaluated in stroma, within tumour scar border, after excluding areas around ductal carcinoma in situ, tumour zones with necrosis and artefacts, and were scored continuously as the average percentage of stromal area occupied by mononuclear cells. PreNAC TILs were described in categories (low: $<10 \%$; intermediate: 11-59\%; high $\geq 60 \%$ ). ${ }^{14}$ Post-NAC TILs were analysed as a continuous variable, as no threshold has yet been determined.

Since RCB and Neo-Bioscore are composed of a different number of risk categories (four risk categories for RCB vs. eight risk categories for Neo-Bioscore, respectively), establishing common risk categories was necessary to compare the two scores. Hence, based on the predicted risk of events, we established three risk categories: low risk (predicted 5-year DFS > 90\%, corresponding to $\mathrm{RCB}-\mathrm{I} / \mathrm{pCR}$ and Neo-Bioscore $0-3$ ); intermediate risk (predicted 5year DFS comprised between 70 and $90 \%$, corresponding to RCB-II and Neo-Bioscore 4-5); and high risk (predicted 5-year DFS $<70 \%$, corresponding to RCB-III and Neo-Bioscore 6-7).

Study endpoints

Disease-free survival (DFS) was defined as the time from surgery to death, loco-regional recurrence or distant recurrence, whichever occurred first. Overall survival (OS) was defined as the time from surgery to death. Patients for whom none of these events were recorded were censored at the date of their last known contact.

Statistical analysis

The Akaike Information Criterion (AIC ${ }^{28}$ was used to compare prognostic performances. AIC determines the best prognostic model from a set of models by selecting the one with the highest likelihood under the constraint of the smallest number of predictors. The lowest AIC value corresponds to the best model.

Discrimination (i.e. whether the relative ranking of individual predictions is in the correct order) was evaluated using the concordance index (C-Index). ${ }^{29}$ C-Index is the probability that given two randomly selected patients, the patient with the most pejorative outcome will in fact have the most pejorative predicted outcome. A C-index value of $0.9-1.0$ corresponds to an excellent discriminative power, whereas a C-Index value of 0.5 corresponds to a worthless test. Its discriminative power is considered poor from 0.6 to 0.7 , fair from 0.7 to 0.8 and good from 0.8 to 0.9 . CIndex can be used for censored data. A bootstrap method with 500 resample was used to determine confidence intervals.

Calibration (i.e. the relationship between outcomes observed and predicted probabilities) was evaluated with graphical representations (calibrations curves). By definition, a wellcalibrated risk score or prediction rule attributes the correct probability of event to all predicted risk levels. A poorly calibrated rule under- or over-predicts the probability of events. Calibration applied on survival analysis are particular since observations are events. Well-calibrated models have an intercept a of zero and a slope $\beta$ of 1 . The calibration of censored data is mainly visual. In this study, 60-months survival was used for the calibration of cox models, which corresponds to our median follow-up.

All analyses were performed in global population and after stratification by BC subtype. Qualitative variables were compared by Chi-square or Fisher exact tests and quantitative ones by Student $t$-tests. Survival probabilities were estimated by Kaplan-Meier method, and survival curves were compared with log-rank tests. Hazard ratios and their 95\% confidence intervals were calculated with the Cox proportional hazards model. Significance threshold was of $5 \%$. Analyses were performed with $\mathrm{R}$ software, version $3.3 .{ }^{30}$

\section{RESULTS}

\section{Patient characteristics}

Our cohort was composed of 750 patients. Patient characteristics are detailed in Appendix Table A1. Mean age at diagnosis was of 48.3 years. Tumour distribution by BC subtype was as follows: luminal: $n=221$ (29.5\%); TNBC: $n=320$ (42.7\%); HER2-positive: $n=209$ (27.9\%). At NAC completion, 281 (37.5\%) patients had reached pCR. After a median follow-up of 52.8 months (Cl [50.2-56.3]), 146 events were observed.

The distribution of patients into the three risk categories established is detailed Table 1. The distribution of RCB and NeoBioscore in global population and by BC subtype is represented in Appendix Fig. A1. 
According to $\mathrm{RCB}, 44.5 \%$ of patients were classified in the lowrisk category ( $37.5 \%$ for RCB-0 and $7.1 \%$ RCB-I, respectively), $38.1 \%$ were classified in the intermediate-risk category and $17.3 \%$ were classified in the high-risk category.

According to Neo-Bioscore, $58.8 \%$ of patients were classified in the low-risk category (Neo-Bioscore 0/1/2/3: 1\%; 8\%; 19\% and 32.7\%, respectively), $40.4 \%$ were classified in the intermediate-risk category, whereas only $0.8 \%$ were classified in the high-risk category.

\begin{tabular}{|c|c|c|c|c|c|}
\hline RCB-0 & $281(37.5)$ & 31 (14) & $138(43.1)$ & $112(53.6)$ & Low (44.5\%) \\
\hline RCB-I & $53(7.1)$ & $14(6.3)$ & $19(5.9)$ & $20(9.6)$ & \\
\hline \multicolumn{6}{|c|}{ Neo-Bioscore } \\
\hline 0 & $5(0.7)$ & $0(0)$ & $0(0)$ & $5(2.4)$ & Low \\
\hline 1 & $54(7.2)$ & $12(5.4)$ & $0(0)$ & $42(20.1)$ & (58.8\%) \\
\hline 2 & 147 (19.6) & $54(24.4)$ & $12(3.8)$ & $81(38.8)$ & \\
\hline 3 & 235 (31.3) & $79(35.7)$ & $96(30)$ & $60(28.7)$ & \\
\hline 7 & $0(0)$ & $0(0)$ & $0(0)$ & $0(0)$ & $(0.8 \%)$ \\
\hline
\end{tabular}

Low-risk category $=$ predicted 5 -year DFS $>90 \%$, Intermediate-risk category $=$ predicted 5 -year DFS comprised between 70 and $90 \%$, High-risk categor $=$ predicted 5-year DFS $<70 \%$.
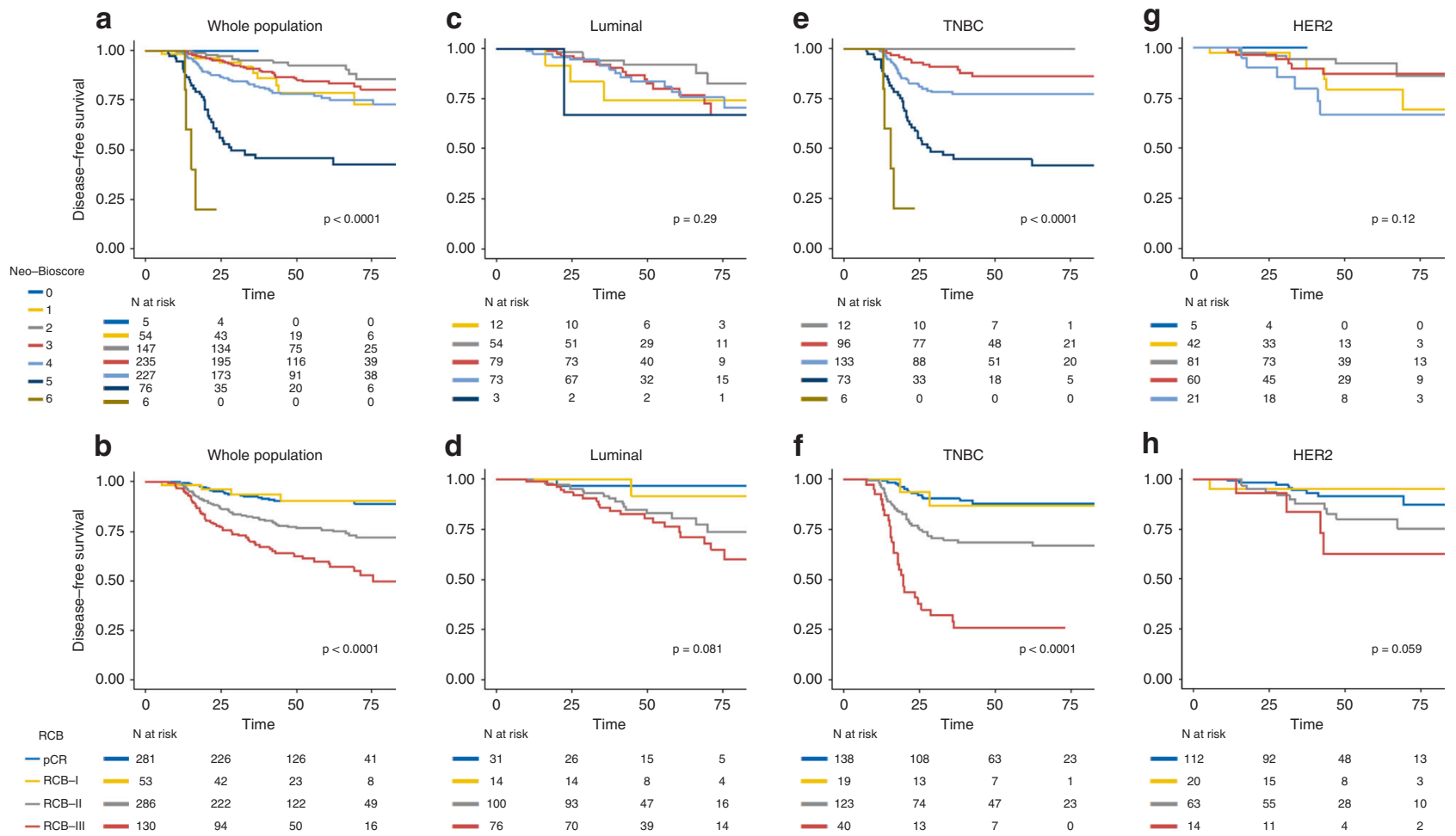

Fig. 1 Association between RCB, Neo-Bioscore and disease free survival in the whole population and by pathological subtypes. a Neobioscore in the whole population; $\mathbf{b}$ RCB in the whole population; $\mathbf{c}$ Neo-bioscore in the Luminal subtype; $\mathbf{d}$ RCB in the Luminal subtype; e Neo-bioscore in the Triple negative subtype; $\mathbf{f}$ RCB in the Triple negative subtype; $\mathbf{g}$ Neo-bioscore in the HER2-positive subtype; $\mathbf{h}$ RCB in the HER2-positive subtype. 


\section{4}

associated to a very good prognosis ( 5 y DFS rate $=90 \%, \mathrm{Cl}$ [86.5-94.4\%] and 90\% Cl [81.7-100], respectively).

Similar results were obtained for OS (Appendix Fig. A2).

Comparison of prognostic performance

Prognostic performance. We assessed the prognostic performance of Neo-Bioscore and RCB by calculating AIC (Fig. 2a-d). In global population, Neo-Bioscore was associated to a slightly lower AIC than RCB (AIC: 1738 vs. 1756, respectively), corresponding to a better prognostic performance.

However, RCB had the lowest AICs for every BC subtype (luminal: 403 vs. 408 , respectively; TNBC: 800 vs. 808 , respectively; HER2-positive BC: 232 vs. 234, respectively).

Discrimination. In global population, $\mathrm{C}$-Index values were poor for RCB $(0.66 ; \mathrm{Cl}[0.61-0.71])$ and fair for Neo-Bioscore $(0.70 ; \mathrm{Cl}$ [0.65-0.75]). C-Index values were higher in TNBC (RCB: $0.73,9 \mathrm{Cl}$ [0.68-0.77]; Neo-Bioscore: $0.71, \mathrm{Cl}$ [0.66-0.75]) and HER2-positive tumours (RCB: 0.64, Cl [0.56-0.73]; Neo-Bioscore: 0.64, Cl [0.56-0.73]) compared to luminal tumours (RCB: $0.61, \mathrm{Cl}$ [0.55-0.67], and Neo-Bioscore: 0.60, Cl [0.53-0.67]) (Fig. 2e-h).

Calibration. Five-year calibration curves are represented Fig. 2i-I. Both Neo-Bioscore and RCB were well calibrated in global population and in every $B C$ subtype. The best calibration was observed for TNBC patients. Altogether, both RCB and Neo-Bioscore were well calibrated among $B C$ subtypes, and accurately discriminated the risk of patients. Neo-Bioscore had good performance in global population. RCB had slightly better prognostic performances when each BC subtype was evaluated separately.

Concordance between Neo-Bioscore and RCB

Distributions of Neo-Bioscore according to RCB, and RCB according to Neo-Bioscore, respectively, are detailed Fig. 3. Concordance between the two scores was poor. $28 \%$ of patients classified RCB-pCR (i.e. low-risk category) corresponded to intermediate and high-risk categories according to NeoBioscore (Neo-Bioscore 4: 23.8\%, and Neo-Bioscore 5: 4.2\%, respectively) (Fig. 3a, c). Conversely, 34.6\% patients classified RCB-III (i.e. high-risk category) corresponded to low-risk categories according to Neo-Bioscore (Neo-Bioscore 2-3: 7.7\% and $26.9 \%$, respectively).

Similarly, among the patients classified in the low-risk categories according to Neo-Bioscore (Neo-Bioscore 0-3), 49.8\% corresponded to intermediate or high-risk categories according to RCB (RCB-II or III) (Fig. 3b, d).

Hence, on an individual scale, RCB and Neo-Bioscore displayed poor consistency (Fig. 3c). RCB and Neo-Bioscore were discrepant in $49.3 \%$ of cases when classifying patients into the three risk categories (low/intermediate/high). Despite good prognostic performances on a population scale, RCB and Neo-Bioscore were poorly concordant on an individual scale.

In BC subtypes, the highest concordance was observed for HER2-positive tumours ( $67 \%$ vs. only $30 \%$ of concordant predictions for luminal tumours) (Appendix Figs. A3, A4). 50.9\% of predictions were concordant for TNBC tumours (Appendix Fig. A5). In the patient with a real event (recurrence, metastasis or death), concordance decreased to $35.5 \%$ in global population, $29.4 \%$ in HER2-positive tumours, and only $16.6 \%$ in the luminal tumours (vs. $48 \%$ in the TNBC population), underlying the difficulty of models to predict survival in high-risk groups.

Addition of histological variables

Adding pathological variables to RCB and Neo-Bioscore slightly improved their prognostic performance.

In global population, AICs of models were systematically improved by adding LVI or pre-/post-NAC TILs level (Fig. 2). The best AIC was observed for Neo-Bioscore + LVI.

When analysing by BC subtype (Fig. 2), LVI also improved AICs of both RCB and Neo-Bioscore for TNBC and luminal tumours. For HER2-positive patients, pre- and post-NAC TILs levels improved models the most. a

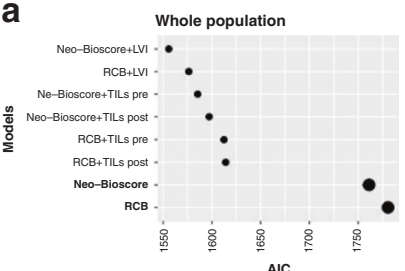

e
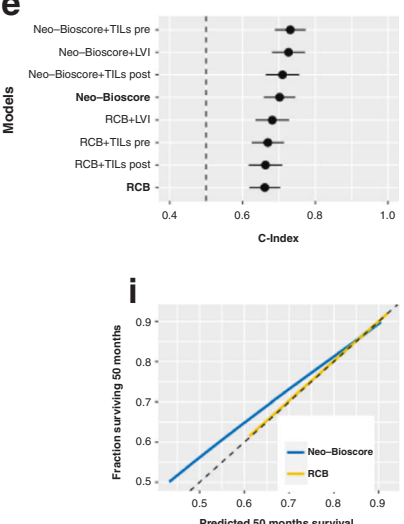

b

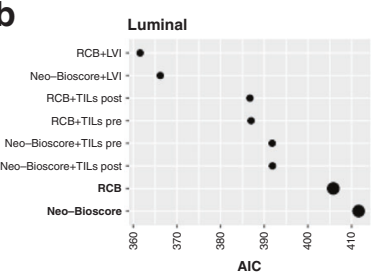

f

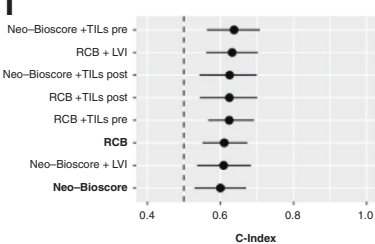

j

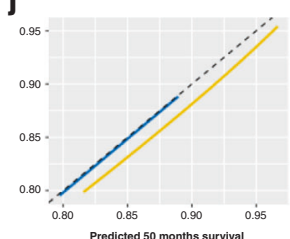

C

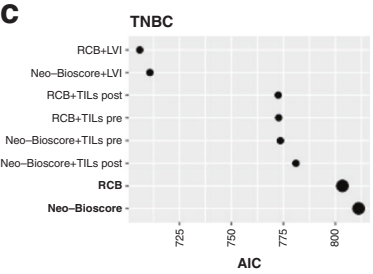

g

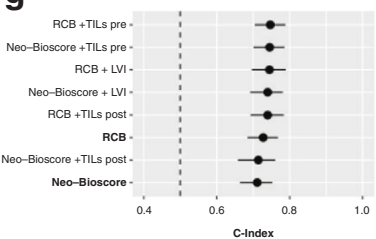

k

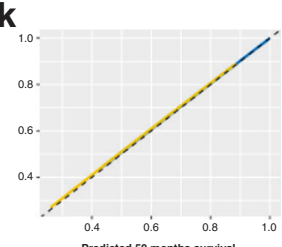

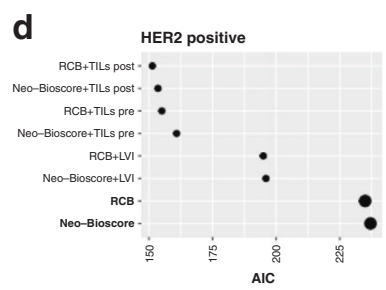

h

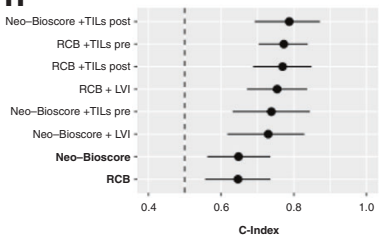

I

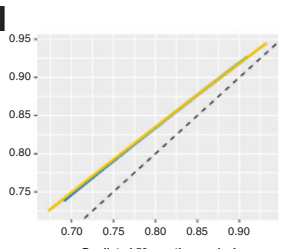

Fig. 2 AIC, C-index and calibration curves for RCB and neo-bioscore, in whole population and by pathological substypes. a AIC in the whole population; b AIC in the luminal subtype; c AIC in the triple negative subtype; d AIC in the HER2-positive subtype; e C-index in the whole population; $\mathbf{f}$ C-index in the luminal subtype; $\mathbf{g}$ C-index in the triple negative subtype; $\mathbf{h}$ C-index in the HER2-positive subtype; i Calibration curves for the whole population; $\mathbf{j}$ Calibration curves for the luminal subtype; $\mathbf{k}$ Calibration curves for the triple negative subtype; I Calibration curves for the HER2-positive subtype. 


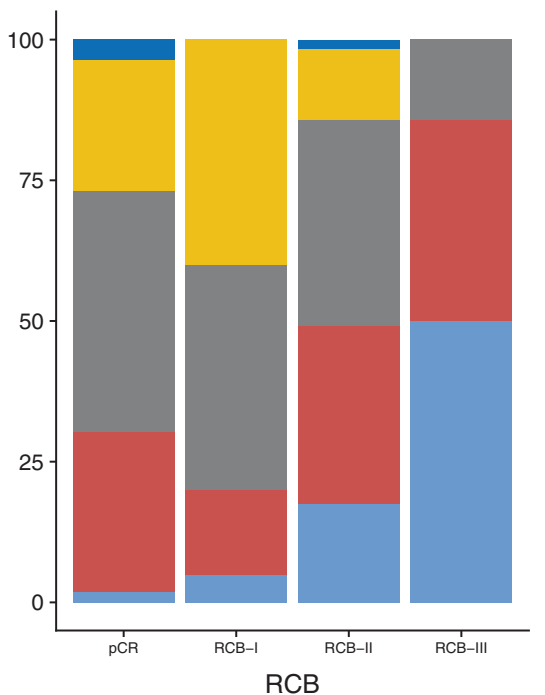

b

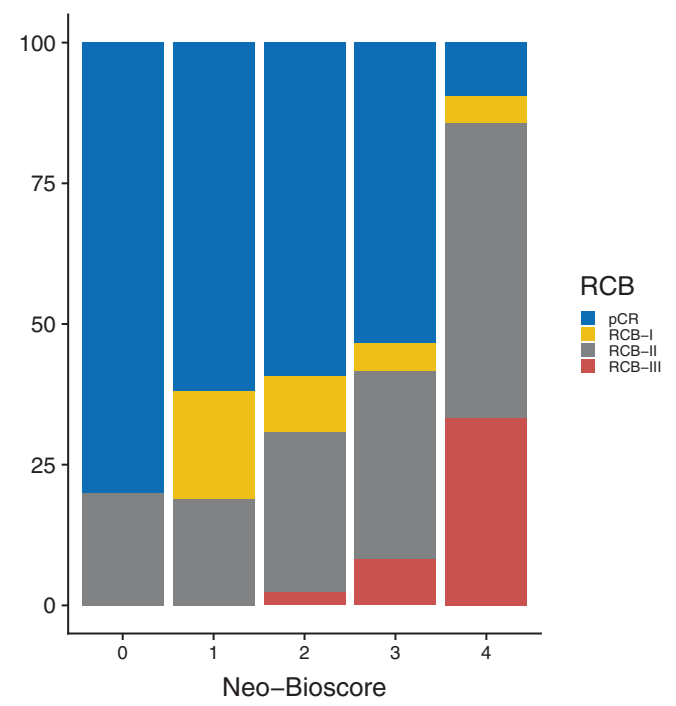

C
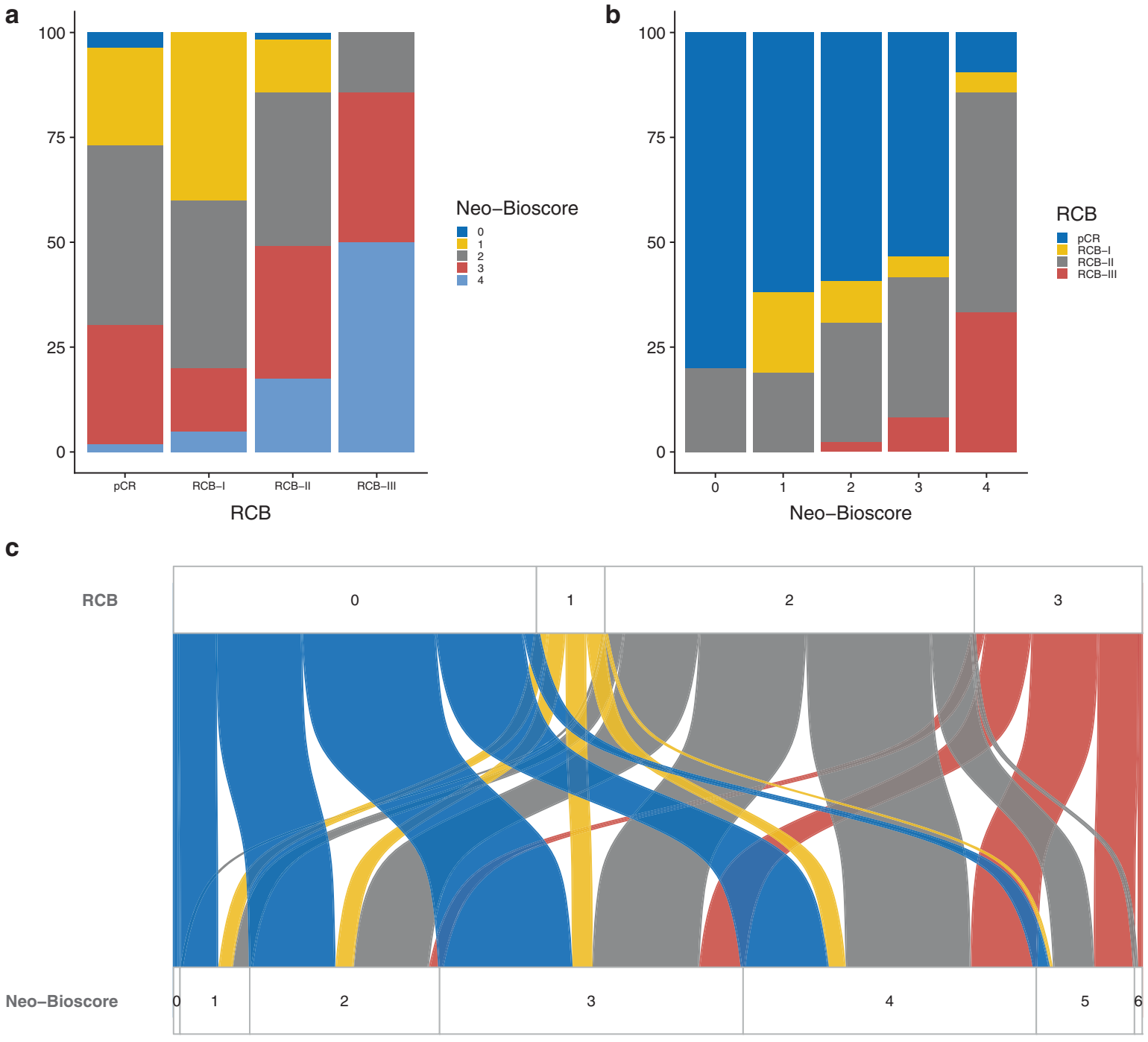

d

Neo-Bioscore

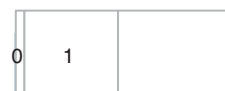

2

3

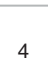

5

$$
6
$$

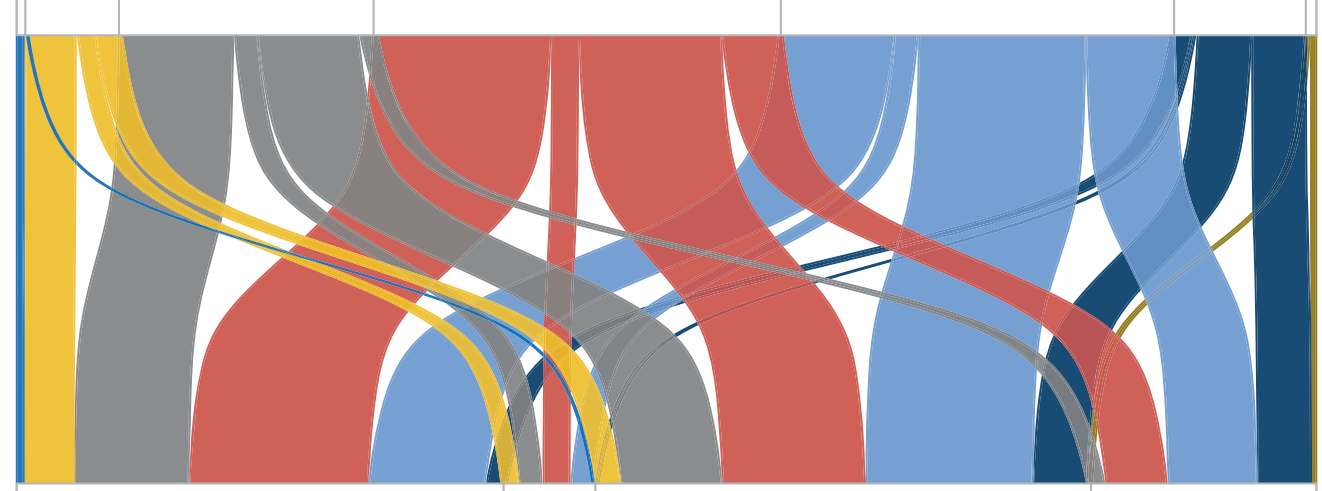

RCB

0

2

3

Fig. 3 Concordance between RCB and neo-bioscore in the global population. a Neo-bioscore repartition according to RCB. b RCB repartition according to neo-bioscore. c Sankey plot of repartition of neo-bioscore according to RCB. $\mathbf{d}$ Sankey plot of repartition of RCB according to neo-bioscore. 
However, adding pathological variables did not improve C-Index, neither in global population, nor in BC subtypes.

\section{DISCUSSION}

Our study on a large cohort of BC patients treated with NAC showed that RCB and Neo-Bioscore were both significantly associated with prognosis in global population and in $\mathrm{BC}$ subtypes, especially for TNBC patients. Analyses were not relevant in the HER2-positive population due to the small number of events. Neo-Bioscore showed better performance in global population, whereas RCB offered better performance in pathological subtypes, notably for luminal and TNBC. In addition, our results suggest that these scores might be improved by the addition of pathological variables such as TILs or LVI. However, on an individual scale, RCB and Neo-Bioscore were highly discrepant in their predictions ( $50 \%$ of consistency between risk categories).

Both scores had been individually validated in independent cohorts. An external validation of Neo-Bioscore was performed by Bergquist et al. $^{31}$ on 43.320 patients from the National Cancer Database. Neo-Bioscore had a greater discriminative power compared to CPS + EG and AJCC clinical staging (5-year OS). The long-term prognostic relevance of $R C B$ within each $B C$ subtype was demonstrated by Symmans et al. ${ }^{32}$ in five BC cohorts $(n=1158)$. RCB has also been shown to be highly reproducible. ${ }^{33}$ Concerning direct comparison of the different scoring systems, Choi et al. ${ }^{34}$ evaluated seven pathologic classification systems, of which RCB and CPS + EG. RCB had the best performance compared to the other scoring systems, both for OS and distant disease-free survival (DDFS). However, Neo-Bioscore (an improvement of the CPS + EG score) was not evaluated in the latter study. Provenzano et al. ${ }^{24}$ had already suggested that RCB index was the preferred method for more detailed quantification of residual disease at NAC completion. Hence, our study adds strength to literature by being the first to our knowledge to compare the prognostic performance of RCB and Neo-Bioscore on a large reallife cohort of BC patients.

Identifying the best model to classify patients into prognostic categories after NAC is crucial, notably since scoring systems can help identify patients of poor prognosis that can be candidates to further second-line treatments or clinical trials. In addition, it appears important to determine the best prognostic score so that a unique score can be used in studies, to help improve their comparability. Indeed, different staging systems yield different estimates for future risks. Very different predictions can be obtained for the same patient. In our study, both scores showed high performance on a population scale, but were poorly concordant with one another on an individual scale. These limitations are common to numerous risk calculators and partially explains the lack of widespread use in routine practice. ${ }^{35}$ These differences could be explained by the fact that these models do not capture the same response patterns. Indeed, whereas RCB only considers tumour size variables (primary tumour dimensions, tumour bed cellularity and axillary nodal burden), Neo-Bioscore also comprises pathological characteristics such as oestrogen-receptor status, nuclear grade and HER2 status. Altogether, since prognostic predictions in global population are currently outdated, pathological subtypes should be considered as distinct entities, and their prognosis should be evaluated independently. Another explanation for these discrepancies could come from the difference (and the very large number) of categories of both models. Hence, the worst concordance rates were found in the patient who expected an event (recurrence, death or metastasis), highlighting the difficulty of numerous models to identify the high-risk groups.

In an effort to improve prognostic performance, other studies suggested considering pathological variables as prognostic elements after NAC, alone or in combination with existing models. ${ }^{13,20,23}$ Von Minckwitz et al. ${ }^{36}$ evaluated Ki67, a proliferation index, as a prognostic marker on 1151 patients after NAC. Patients with high Ki67 levels (>35.1) had significantly higher recurrence and death rates compared to patients with low or intermediate Ki67 levels. Adding Ki67 to the analysis of $\mathrm{pCR}$ was more contributive than pCR only for luminal BC. Sheri et al. ${ }^{37}$ also showed that the addition of post-treatment Ki67 to RCB improved the prediction of long-term outcome. However, Ki67 suffers from poor reproducibility and its assessment is difficult to standardise. ${ }^{38}$ Hence, its clinical utility remains limited in routine care. TILs on pretreatment biopsy have been associated with high $\mathrm{pCR}$ rates in the neoadjuvant setting and with better outcomes in the adjuvant setting. ${ }^{14,20,21,23}$ In addition, their assessment is rather standardised according to guidelines of the TILs working group. ${ }^{27}$ The added value of post-NAC TILs remains to be determined. Indeed, different prognostic values have been described among $B C$ subtypes, with higher levels being associated with a good outcome in TNBC patients with residual disease after $\mathrm{NAC}^{39}$ but with a poor outcome in HER2-positive patients. ${ }^{23}$ Asano et al. ${ }^{40}$ suggested that RCB combined with post-NAC TILs could be a more sensitive predictor for $B C$ recurrence after NAC, with a 0.048 risk of recurrence (hazard ratio) in global population for RCB-TILs-positive patients $(p<0.001), 0.041$ in TNBC patients $(p=0.018), 0.134$ in HER2-positive patients $(p=0.036)$ and 0.081 for luminal tumours $(p=0.002)$. Future models should probably integrate this information.

In conclusion, our results suggest that although RCB and NeoBioscore offer similar prognostic performances to classify patients at NAC completion in global population, RCB showed better performance in pathological subtypes, especially in luminal and TNBC. RCB offers the advantage of generating fewer prognostic classes compared to Neo-Bioscore, which enables an easier use in everyday real-life practice. Further studies are warranted to confirm the present data and to evaluate the prognostic performance of other pathological variables like TILs or LVI that could be included in future scores to improve their prognosis performance.

\section{ACKNOWLEDGEMENTS}

None.

\section{AUTHOR CONTRIBUTIONS}

Wrote the paper: J.L., E.L. Proofreading English text: J.L. Conceived and designed the experiments: E.L., A.S.H., G.B., D.d.C., J.Y.P., F.R. Analysed the data: E.L., J.L., T.B., J.G., M.L., J.G.F., F.C. Contributed to materials/analysis tools: D.d.C., J.G.F., F.C.

\section{ADDITIONAL INFORMATION}

Ethics approval and consent to participate Approved by the Breast Cancer Study Group of Institut Curie, the study was conducted according to institutional and ethical rules concerning research on tissue specimens and patients and in accordance with the Declaration of Helsinki. Informed consent from patients was not required.

Data availability All supplementary data are available from authors on reasonable request.

Competing interests The authors declare no competing interests.

Funding information None.

Supplementary information is available for this paper at https://doi.org/10.1038/ s41416-020-01251-3.

Note This work is published under the standard license to publish agreement. After 12 months the work will become freely available and the license terms will switch to a Creative Commons Attribution 4.0 International (CC BY 4.0). 
Publisher's note Springer Nature remains neutral with regard to jurisdictional claims in published maps and institutional affiliations.

\section{REFERENCES}

1. Kaufmann, M., von Minckwitz, G., Smith, R., Valero, V., Gianni, L., Eiermann, W. et al. International expert panel on the use of primary (Preoperative) systemic treatment of operable breast cancer: review and recommendations. J. Clin. Oncol. 21, 2600-2608 (2003).

2. Kaufmann, M., von Minckwitz, G., Bear, H. D., Buzdar, A., McGale, P., Bonnefoi, H. et al. Recommendations from an international expert panel on the use of neoadjuvant (primary) systemic treatment of operable breast cancer: new perspectives 2006. Ann. Oncol. 18, 1927-1934 (2007).

3. Mieog, J. S. D., van der Hage, J. A. \& van de Velde, C. J. H. Neoadjuvant chemotherapy for operable breast cancer. Br. J. Surg. 94, 1189-1200 (2007).

4. Mauri, D., Pavlidis, N. \& loannidis, J. P. A. Neoadjuvant versus adjuvant systemic treatment in breast cancer: a meta-analysis. J. Natl Cancer Inst. 97, 188-194 (2005).

5. Jackisch, C., Harbeck, N., Huober, J., von Minckwitz, G., Gerber, B., Kreipe, H.-H. et al. 14th St. Gallen International Breast Cancer Conference 2015: Evidence, Controversies, Consensus-Primary Therapy of Early Breast Cancer: Opinions Expressed by German Experts. Breast Care Basel Switz. 10, 211-219 (2015).

6. Brandão, M., Reyal, F., Hamy, A.-S. \& Piccart-Gebhart, M. Neoadjuvant treatment for intermediate/high-risk HER2-positive and triple-negative breast cancers: no longer an « option » but an ethical obligation. ESMO Open 4, e000515 (2019).

7. Reyal, F., Hamy, A. S. \& Piccart, M. J. Neoadjuvant treatment: the future of patients with breast cancer. ESMO Open 3, e000371 (2018).

8. Symmans, W. F., Peintinger, F., Hatzis, C., Rajan, R., Kuerer, H., Valero, V. et al. Measurement of residual breast cancer burden to predict survival after neoadjuvant chemotherapy. J. Clin. Oncol. 25, 4414-4422 (2007).

9. Guarneri, V., Broglio, K., Kau, S.-W., Cristofanilli, M., Buzdar, A. U., Valero, V. et al. Prognostic value of pathologic complete response after primary chemotherapy in relation to hormone receptor status and other factors. J. Clin. Oncol. 24, 1037-1044 (2006).

10. Cortazar, P., Zhang, L., Untch, M., Mehta, K., Costantino, J. P., Wolmark, N. et al. Pathological complete response and long-term clinical benefit in breast cancer: the CTNeoBC pooled analysis. Lancet 384, 164-172 (2014).

11. Jeruss, J. S., Mittendorf, E. A., Tucker, S. L., Gonzalez-Angulo, A. M., Buchholz, T. A., Sahin, A. A. et al. Combined use of clinical and pathologic staging variables to define outcomes for breast cancer patients treated with neoadjuvant therapy. J. Clin. Oncol. 26, 246-252 (2008).

12. Mittendorf, E. A., Vila, J., Tucker, S. L., Chavez-MacGregor, M., Smith, B. D., Symmans, W. F. et al. The Neo-Bioscore update for staging breast cancer treated with neoadjuvant chemotherapy: incorporation of prognostic biologic factors into staging after treatment. JAMA Oncol. 2, 929-36 (2016).

13. Hamy, A.-S., Lam, G.-T., Laas, E., Darrigues, L., Balezeau, T., Guerin, J. et al. Lymphovascular invasion after neoadjuvant chemotherapy is strongly associated with poor prognosis in breast carcinoma. Breast Cancer Res. Treat. 169, 295-304 (2018).

14. Denkert, C., Minckwitz, G., von, Darb-Esfahani, S., Lederer, B., Heppner, B. I., Weber, K. E. et al. Tumour-infiltrating lymphocytes and prognosis in different subtypes of breast cancer: a pooled analysis of 3771 patients treated with neoadjuvant therapy. Lancet Oncol. 19, 40-50 (2018).

15. Lee, A. H. S., Pinder, S. E., Macmillan, R. D., Mitchell, M., Ellis, I. O., Elston, C. W. et al. Prognostic value of lymphovascular invasion in women with lymph node negative invasive breast carcinoma. Eur. J. Cancer 42, 357-362 (2006).

16. Rakha, E. A., Martin, S., Lee, A. H. S., Morgan, D., Pharoah, P. D. P., Hodi, Z. et al. The prognostic significance of lymphovascular invasion in invasive breast carcinoma. Cancer 118, 3670-3680 (2012).

17. Liu, Y. L., Saraf, A., Lee, S. M., Zhong, X., Hibshoosh, H., Kalinsky, K. et al. Lymphovascular invasion is an independent predictor of survival in breast cancer after neoadjuvant chemotherapy. Breast Cancer Res. Treat. 157, 555-564 (2016).

18. Choi, M. K., Park, Y. H., Kil, W. H., Lee, J. E., Nam, S. J., Ahn, J. S. et al. Clinicopathological features of early failure of neoadjuvant chemotherapy in locally advanced breast cancer. Cancer Chemother. Pharmacol. 74, 521-529 (2014).

19. Abdel-Fatah, T. M., Ball, G., Lee, A. H. S., Pinder, S., MacMilan, R. D., Cornford, E. et al. Nottingham Clinico-Pathological Response Index (NPRI) after neoadjuvant chemotherapy (Neo-ACT) accurately predicts clinical outcome in locally advanced breast cancer. Clin. Cancer Res. 21, 1052-1062 (2015).
20. Mao, Y., Qu, Q., Zhang, Y., Liu, J., Chen, X. \& Shen, K. The value of tumor infiltrating lymphocytes (TILs) for predicting response to neoadjuvant chemotherapy in breast cancer: a systematic review and meta-analysis. PLoS ONE 9, e115103 (2014).

21. Denkert, C., Loibl, S., Noske, A., Roller, M., Müller, B. M., Komor, M. et al. Tumorassociated lymphocytes as an independent predictor of response to neoadjuvant chemotherapy in breast cancer. J. Clin. Oncol. 28, 105-113 (2010).

22. Denkert, C., von Minckwitz, G., Brase, J. C., Sinn, B. V., Gade, S., Kronenwett, R. et al. Tumor-infiltrating lymphocytes and response to neoadjuvant chemotherapy with or without carboplatin in human epidermal growth factor receptor 2positive and triple-negative primary breast cancers. J. Clin. Oncol. 33, 983-991 (2015).

23. Hamy, A.-S., Pierga, J.-Y., Sabaila, A., Laas, E., Bonsang-Kitzis, H., Laurent, C. et al. Stromal lymphocyte infiltration after neoadjuvant chemotherapy is associated with aggressive residual disease and lower disease-free survival in HER2-positive breast cancer. Ann. Oncol. 28, 2233-2240 (2017).

24. Provenzano, E., Bossuyt, V., Viale, G., Cameron, D., Badve, S., Denkert, C. et al. Standardization of pathologic evaluation and reporting of postneoadjuvant specimens in clinical trials of breast cancer: recommendations from an international working group. Mod. Pathol. 28, 1185-1201 (2015).

25. Residual Cancer Burden Calculator [Internet]. http://www3.mdanderson.org/app/ medcalc/index.cfm?pagename $=$ jsconvert $3 \mathrm{n}$ (2018).

26. Giuliano, A. E., Connolly, J. L., Edge, S. B., Mittendorf, E. A., Rugo, H. S., Solin, L. J. et al. Breast Cancer-Major changes in the American Joint Committee on Cancer eighth edition cancer staging manual. CA Cancer J. Clin. 67, 290-303 (2017).

27. Salgado, R., Denkert, C., Demaria, S., Sirtaine, N., Klauschen, F., Pruneri, G. et al. The evaluation of tumor-infiltrating lymphocytes (TILs) in breast cancer: recommendations by an International TILs Working Group 2014. Ann. Oncol. 26, 259-271 (2015).

28. Akaike, H. A new look at the statistical model identification. IEEE Trans. Autom. Control 19, 716-723 (1974).

29. Harrell, F. E., Lee, K. L. \& Mark, D. B. Multivariable prognostic models: issues in developing models, evaluating assumptions and adequacy, and measuring and reducing errors. Stat. Med. 15, 361-387 (1996).

30. $R$ : The $R$ Project for Statistical Computing [Internet]. https://www.r-project.org/. (2018).

31. Bergquist, J. R., Murphy, B. L., Storlie, C. B., Habermann, E. B. \& Boughey, J. C. Incorporation of treatment response, tumor grade and receptor status improves staging quality in breast cancer patients treated with neoadjuvant chemotherapy. Ann. Surg. Oncol. 24, 3510-3517 (2017).

32. Symmans, W. F., Wei, C., Gould, R., Yu, X., Zhang, Y., Liu, M. et al. Long-term prognostic risk after neoadjuvant chemotherapy associated with residual cancer burden and breast cancer subtype. J. Clin. Oncol. 35, 1049-1060 (2017).

33. Peintinger, F., Sinn, B., Hatzis, C., Albarracin, C., Downs-Kelly, E., Morkowski, J. et al. Reproducibility of residual cancer burden for prognostic assessment of breast cancer after neoadjuvant chemotherapy. Mod. Pathol. 28, 913-920 (2015).

34. Choi, M., Park, Y. H., Ahn, J. S., Im, Y.-H., Nam, S. J., Cho, S. Y. et al. Assessment of pathologic response and long-term outcome in locally advanced breast cancers after neoadjuvant chemotherapy: comparison of pathologic classification systems. Breast Cancer Res. Treat. 160, 475-489 (2016).

35. Laas, E., Mallon, P., Delomenie, M., Gardeux, V., Pierga, J.-Y., Cottu, P. et al. Are we able to predict survival in ER-positive HER2-negative breast cancer? A comparison of web-based models. Br. J. Cancer 112, 912-917 (2015).

36. von Minckwitz, G., Schmitt, W. D., Loibl, S., Müller, B. M., Blohmer, J. U., Sinn, B. V. et al. Ki67 measured after neoadjuvant chemotherapy for primary breast cancer. Clin. Cancer Res. 19, 4521-4531 (2013).

37. Sheri, A., Smith, I. E., Johnston, S. R., A'Hern, R., Nerurkar, A., Jones, R. L. et al. Residual proliferative cancer burden to predict long-term outcome following neoadjuvant chemotherapy. Ann. Oncol. 26, 75-80 (2015).

38. Focke, C. M., Bürger, H., van Diest, P. J., Finsterbusch, K., Gläser, D., Korsching, E. et al. Interlaboratory variability of Ki67 staining in breast cancer. Eur. J. Cancer 84, 219-227 (2017).

39. Dieci, M. V., Criscitiello, C., Goubar, A., Viale, G., Conte, P., Guarneri, V. et al. Prognostic value of tumor-infiltrating lymphocytes on residual disease after primary chemotherapy for triple-negative breast cancer: a retrospective multicenter study. Ann. Oncol. 25, 611-618 (2014).

40. Asano, Y., Kashiwagi, S., Goto, W., Takada, K., Takahashi, K., Hatano, T. et al. Prediction of survival after neoadjuvant chemotherapy for breast cancer by evaluation of tumor-infiltrating lymphocytes and residual cancer burden. BMC Cancer 17, 888 (2017). 